\title{
Velocity and the variability of anticipated and unanticipated money growth in Malaysia
}

\begin{abstract}
This paper investigates the causal linkages between velocity of money and both anticipated and unanticipated components of money supply growth, and its variability as measured on the Malaysian annual data (1973 94). The motivation is to provide evidence in a developing country context about whether the authorities should pay attention to signals from anticipated or unanticipated or both components of money supply aggregate. The empirical findings from the VAR model support the hypothesis not only that the components of money supply growth Granger-cause the growth of money velocity but also in its variability measures offer an additional evidence.
\end{abstract}

Keyword: Velocity of money; Money supply growth 